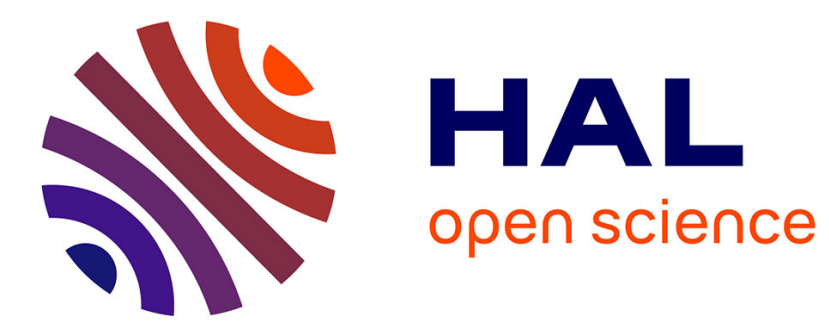

\title{
Domain Decomposition Method using Integral Equations for Studying Antenna Sitting on Large Platforms
}

\author{
J. Maurin, A. Barka, V. Gobin, B. Gabard
}

\section{To cite this version:}

J. Maurin, A. Barka, V. Gobin, B. Gabard. Domain Decomposition Method using Integral Equations for Studying Antenna Sitting on Large Platforms. 2014 IEEE International Conference on Antenna Measurements \& Applications, Nov 2014, ANTIBES, France. hal-01087749

\section{HAL Id: hal-01087749 \\ https://hal.science/hal-01087749}

Submitted on 26 Nov 2014

HAL is a multi-disciplinary open access archive for the deposit and dissemination of scientific research documents, whether they are published or not. The documents may come from teaching and research institutions in France or abroad, or from public or private research centers.
L'archive ouverte pluridisciplinaire HAL, est destinée au dépôt et à la diffusion de documents scientifiques de niveau recherche, publiés ou non, émanant des établissements d'enseignement et de recherche français ou étrangers, des laboratoires publics ou privés. 


\title{
Domain Decomposition Method using Integral Equations for Studying Antenna Sitting on Large Platforms
}

\author{
J. Maurin, A. Barka, V. Gobin, B. Gabard \\ Electromagnetism and Radar Department \\ ONERA \\ Toulouse, France \\ julien.maurin@onera.fr, andre.barka@onera.fr, vincent.gobin@onera.fr, benjamin.gabard@onera.fr
}

\begin{abstract}
In this paper, we present a non-overlapping domain decomposition method using integral equations in the frequency domain for the resolution of wave scattering from metallic objects. The objective is to use multi-domain modelling to process multi-scale structures. This method is flexible thanks to an independance between sub-domains and relying on the local resolution of these sub-domains. Moreover, the convergence is improved compared to a classical resolution. This method is achieved for predicting radar cross-sections, studying antenna sitting on large platforms and EMC applications.
\end{abstract}

\section{INTRODUCTION}

This paper deals with a computational method developed to combine domain decomposition and integral equations. The resolution of the Maxwell equations by integral equations is very useful to study the wave scattering from non-penetrable objects or homogeneous objects represented by a surface impedance. These methods present the advantage to generate a surfacic mesh of the object which is better than a volumic mesh as in the finite element methods. However, these methods lead to a dense linear system.

Combining a multi-domain approach with integral equations should permit to solve some problems we meet with a classic mono-domain approach. Such a method has been proposed in [1]. Nevertheless, the formulation does not permit to solve opened structures and a random meshing can accidentelly lead to a singular linear system. In this paper, we present a more general formulation correcting these problems. The resolution of the linear system relies on a local resolution of the subdomains.

\section{Mono-Domain InTEgRAL EQUATIONS}

The total electromagnetic field $(\mathbf{E}, \mathbf{H})$ of frequency $f$ can be decomposed in an incident field $\left(\mathbf{E}^{\text {source }}, \mathbf{H}^{\text {source }}\right)$ and the field $\left(\mathbf{E}^{\mathrm{sc}}, \mathbf{H}^{\mathrm{sc}}\right)$ scattered by the object. The studied PEC (Perfectly Electric Conductor) object takes up the domain $\Omega$ with the boundary $\Gamma$ of exterior normal $\hat{\mathbf{n}}$. Solving integral equations on a PEC object consists in computing electric currents $\mathbf{j}=$ $\hat{\mathbf{n}} \times \mathbf{H}$ which are the tangential traces of the magnetic field. The fields scattered by the structure can be computed knowing the electric currents on $\Gamma$.
From the Stratton-Chu formulae [2] and by application of the method of moments, the Electric Field Integral Equations (EFIE) and Magnetic Field Integral Equations (MFIE) can be developed. The scalar product $\langle\mathbf{a}, \mathbf{b}\rangle_{\Gamma}=\int_{\Gamma} \mathbf{a} \cdot \mathbf{b} \mathrm{d} \Gamma$ and the test and basis Rao-Wilton-Glisson (RWG) functions $\Phi \in H_{\text {div }}(\Gamma)$ are defined [3].

This is the EFIE with our operator $(B-S)$ [4]:

$$
\langle(B-S)(\mathbf{j}, \Gamma), \boldsymbol{\Phi}\rangle_{\Gamma}=\left\langle I \mathbf{E}^{\text {source }}, \boldsymbol{\Phi}\right\rangle_{\Gamma}
$$

This is the MFIE with our operator $\left(\frac{I}{2}+\imath Q^{\times}\right)[4]$ :

$$
\left\langle\left(\frac{I}{2}+\imath Q^{\times}\right) \mathbf{j}, \boldsymbol{\Phi}\right\rangle_{\Gamma}=\left\langle I \hat{\mathbf{n}} \times \mathbf{H}^{\text {source }}, \boldsymbol{\Phi}\right\rangle_{\Gamma}
$$

The EFIE and MFIE can suffer of internal resonances and a solution is the Combined Field Integral Equations which consists in combining EFIE and MFIE such as CFIE = $\alpha E F I E+(1-\alpha) M F I E$ with $\alpha \in] 0 ; 1[$.

\section{Multi-Domain Integral Equations}

Consider a PEC object $\Omega$ divided in two non-overlapping sub-domains such as $\Omega=\Omega_{1} \cup \Omega_{2}$. The boundaries of these domains are noted $\Gamma, \Gamma_{1}$ and $\Gamma_{2}$. The normal vectors $\hat{\mathbf{n}}, \hat{\mathbf{n}}_{1}$ and $\hat{\mathbf{n}}_{2}$ are exterior to the surfaces. Note that all the surfaces of the sub-domains are PEC. The surfaces $\Gamma_{1}^{i}=\Gamma_{1} \cap \Gamma_{2}$ and $\Gamma_{2}^{i}=\Gamma_{2} \cap \Gamma_{1}$ which did not exist in the original mono-domain problem are called interfaces. They are the junction between two sub-domains. The exterior boundaries which exist in the original problem are noted $\Gamma_{1}^{b}$ and $\Gamma_{2}^{b}$. This decomposition is pictured in Fig. 1. The decomposition in nine domains of a F100 aircraft is presented in Fig. 2

\section{A. Formulation}

Two sets of equations permit to develop the Integral Equations - Domain Decomposition Method (IE-DDM) formulation. The first set is the CFIE applied to each sub-domain and the second set is a coupling equation linking sub-domains to each other. The following formulation is exposed for an object decomposed in two sub-domains but it can be extended to any decomposition. 


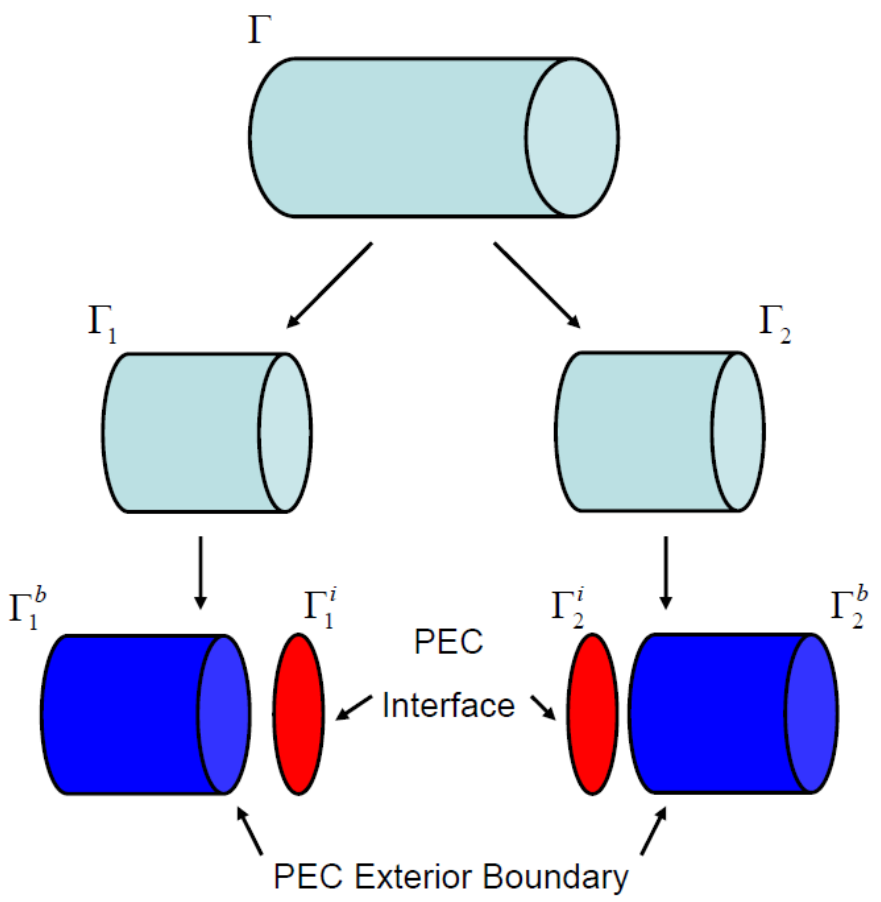

Fig. 1. Description of the domain decomposition

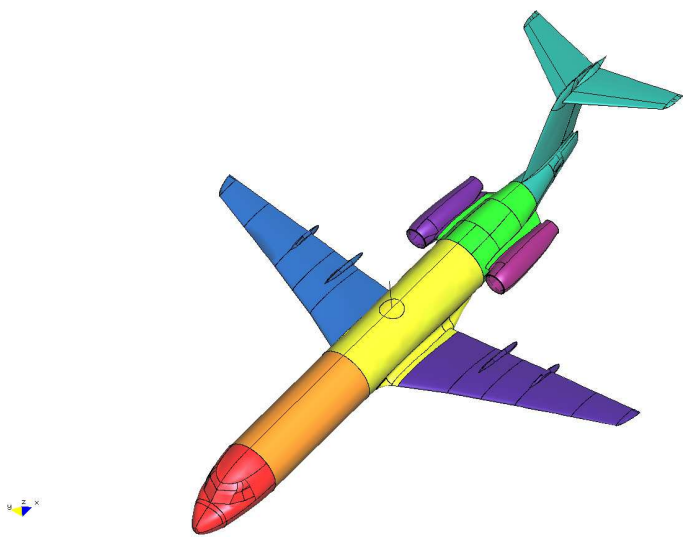

Fig. 2. Practical domain decomposition of a real aircraft

The CFIE on one sub-domain describes the total field on the structure which is the sum of the incident field on the sub-domain with the field scattered by the sub-domain. The coupling equations describe that the incident wave is not the only source that illuminates the first sub-domain. Indeed, the field scattered by the second sub-domain is like a second source for the first sub-domain.

MFIE formulation leads to a simplified form on the interfaces $\Gamma_{1}^{i}$ and $\Gamma_{2}^{i}$. which renders the opposition of currents $\mathbf{j}_{1}$ and $\mathbf{j}_{2}$ on this surface. Consequently, the EFIE formulation is the same on each point of the sub-domain, but we modify the MFIE formulation on the interface. Finally, compared to [1], we propose a new formulation allowing both EFIE, MFIE and CFIE resolutions. Consider the first sub-domain, the weak formulation on the exterior boundary $\Gamma_{1}^{b}$ is:

$$
\left\{\begin{array}{l}
\left\langle(B-S)\left(\mathbf{j}_{1}, \Gamma_{1}\right), \boldsymbol{\Phi}\right\rangle_{\Gamma_{1}^{b}}+\left\langle(B-S)\left(\mathbf{j}_{2}, \Gamma_{2}\right), \boldsymbol{\Phi}\right\rangle_{\Gamma_{1}^{b}} \\
=\left\langle I \mathbf{E}_{\mathbf{t g}_{1} \text { source }}, \boldsymbol{\Phi}\right\rangle_{\Gamma_{1}^{b}} \\
\left\langle\left(\frac{I}{2}+\imath Q^{\times}\right)\left(\mathbf{j}_{1}, \Gamma_{1}\right), \boldsymbol{\Phi}\right\rangle_{\Gamma_{1}^{b}} \\
+\left\langle\left(\frac{I}{2}+\imath Q^{\times}\right)\left(\mathbf{j}_{2}, \Gamma_{2}\right), \boldsymbol{\Phi}\right\rangle_{\Gamma_{1}^{b}}=\left\langle I \hat{\mathbf{n}}_{1} \times \mathbf{H}_{1}^{\text {source }}, \boldsymbol{\Phi}\right\rangle_{\Gamma_{1}^{b}}
\end{array}\right.
$$

On the interface $\Gamma_{1}^{i}$ :

$$
\left\{\begin{array}{l}
\left\langle(B-S)\left(\mathbf{j}_{1}, \Gamma_{1}\right), \boldsymbol{\Phi}\right\rangle_{\Gamma_{1}^{i}}+\left\langle(B-S)\left(\mathbf{j}_{2}, \Gamma_{2}\right), \boldsymbol{\Phi}\right\rangle_{\Gamma_{1}^{i}} \\
=\left\langle I \mathbf{E}_{\mathbf{t g}} \text { source }, \boldsymbol{\Phi}\right\rangle_{\Gamma_{1}^{i}} \\
\left\langle I \mathbf{j}_{1}+I \mathbf{j}_{2}, \boldsymbol{\Phi}\right\rangle_{\Gamma_{1}^{i}}=0
\end{array}\right.
$$

The matrices are compressed with the ACA (Adaptive Cross Approximation) algorithm [5]. The hierarchical matrices [6] of the local CFIE matrices are built. Each block of the coupling matrix is either fully assembled or either compressed depending on the distance criterion between sub-domains. The ACA algorithm and its implementation in the proposed methodology are not detailed in this paper.

\section{B. Linear system resolution}

The linear system to solve is $\left(\mathbf{A}_{l}+\mathbf{A}_{c}\right) \mathbf{x}=\mathbf{b}$. $\mathbf{A}_{l}$ is a block matrix where the $\mathrm{k}^{\text {th }}$ block is the local CFIE matrix of the $\mathrm{k}^{\text {th }}$ sub-domain. $\mathbf{A}_{c}$ is a coupling matrix linking subdomains to each other. $\mathbf{b}$ is the right-hand side representing the incident wave. Practically, the linear system is preconditionned by $\mathbf{A}_{l}$, thus $\left(\mathbf{I}+\mathbf{A}_{l}^{-1} \mathbf{A}_{c}\right) \mathbf{x}=\mathbf{A}_{l}^{-1} \mathbf{b}$ is solved. This operation consists in solving local sub-domains rather than the global object. At each global iteration, the local subdomains are solved. The iterative algorithm GCR (Generalized Conjugate Residual) [7] is used to solve the systems. The ACA (Adaptive Cross Approximation) algorithm previously evoked permits to accelerate the matrix-vector products during the resolution.

\section{Numerical Results}

This algorithm was implemented in a program named IE-DDM. The aim of these results is to validate the precision of the IE-DDM method and to prove that the conditionning of the system is improved. The IE-DDM results are compared with the mono-domain integral equations results implemented in the ELSEM3D software developed by ONERA/DEMR [8]. We compare the norm of the currents on the structure, a RCS (Radar Cross Section), a radiation pattern and the convergence of the iterative solver.

\section{A. Diffraction of a plane wave}

The test concerns the diffraction of a wave by a Fokker F100 PEC aircraft decomposed in nine sub-domains. The decomposition is pictured in Fig. 2 The mono-domain aircraft is meshed with 93510 degrees of freedom and the multidomain aircraft is meshed with 76995 degrees of freedom. The number of degrees of freedom of the sub-domains are 
indicated in Tab. I

The incident wave has a frequency of $f=1 \mathrm{GHz}$, is polarized along the $\hat{\mathbf{x}}$ axis and illuminates the scatterer from the $\hat{\mathbf{z}}$ axis. The sub-domain meshes are totally independent from each others.

The first results permit to evaluate the precision of the method. The convergence criterion of the iterative solver is fixed at $\varepsilon=10^{-4}$ for both mono-domain and multi-domain resolutions. A vizualization of the norm of the currents is represented in Fig. 4. This allows to assess quantitatively the method. The bistatic RCS in the zy plane is plotted in Fig. 5. This is a better qualitative indicator on the precision. We can observe a good agreement between the two curves. There is a small shift which can be explained by a difference of the step size of the meshes in certain regions.

Finally, we compare the convergence of the iterative resolutions by ELSEM3D and IE-DDM with a convergence criterion $\varepsilon=10^{-4}$ in Fig. 6. We note that the IE-DDM program converges despite a severe convergence criterion while the mono-domain resolution ELSEM3D fails to converge after 1000 iterations. We conclude that the IE-DDM formulation is a good preconditionner.

We want to give some information about the computational costs. The ACA algorithm permitting to compress matrices and accelerating the matrix-vector products is a work in progress version. That means the code is not optimized especially concerning the computational times. Consequently, we cannot give precise information about these computational costs.

\begin{tabular}{|c|c|}
\hline & Number of degrees of freedom \\
\hline Mono-domain & 93510 \\
\hline Domain 1 & 4698 \\
\hline Domain 2 & 2760 \\
\hline Domain 3 & 13413 \\
\hline Domain 4 & 3219 \\
\hline Domain 5 & 18750 \\
\hline Domain 6 & 11847 \\
\hline Domain 7 & 11937 \\
\hline Domain 8 & 5187 \\
\hline Domain 9 & 5184 \\
\hline Total multi-domain & 76995 \\
\hline
\end{tabular}

TABLE I

MESHES NUMBER OF DEGREES OF FREEDOM

\section{B. Radiation of a monopole antenna}

The second test concerns the radiation of a monopole antenna installed on a PEC Fokker 100 (Fig. 7). The length of the antenna is $\lambda / 4$ for the frequency $f=480 \mathrm{MHz}$. The antenna is fed by a voltage generator of tension $V_{g}=1 \mathrm{~V}$ and of internal impedance $Z_{g}=50 \Omega$. We present in Fig. 8 the radiation pattern of this antenna in the zix plane (pitch plane).

We observe that the radiation pattern got by IE-DDM resolution are close to the radiation pattern got by a standard mono-domain resolution. The ripples on the radiation pattern are due to the multi path effects between the monopole antenna and the wings, engines and tail of the aircraft.

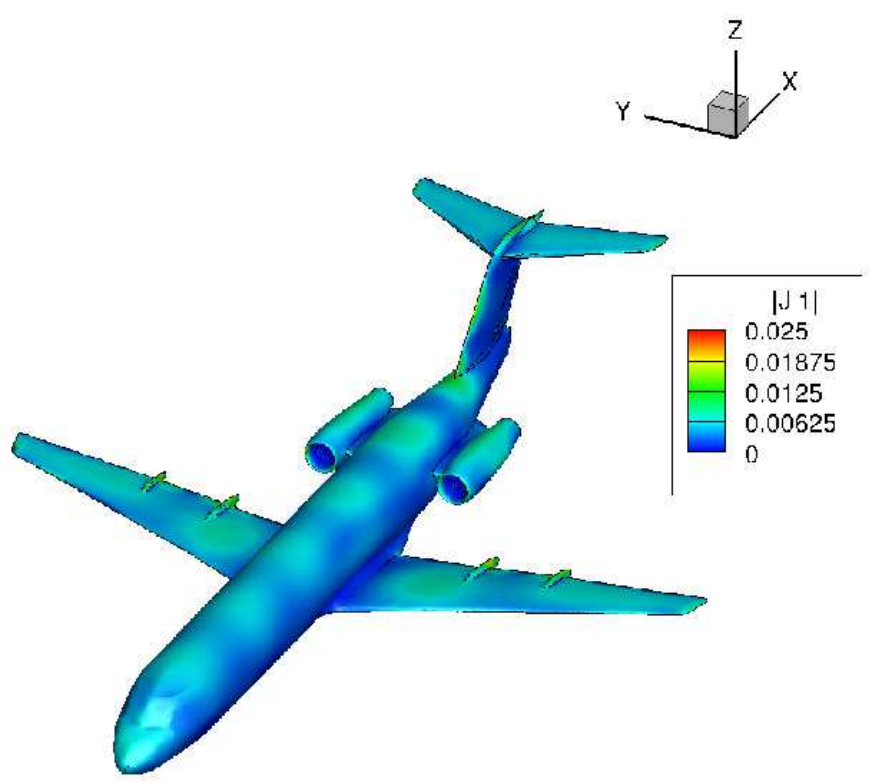

Fig. 3. Electric currents on a PEC F100 by ELSEM3D

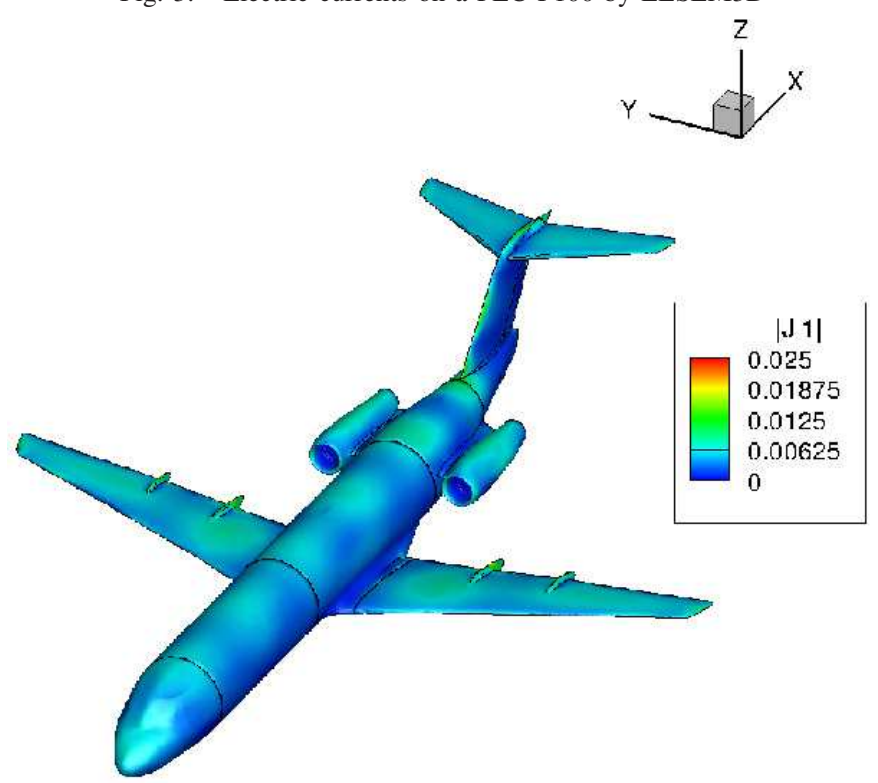

Fig. 4. Electric currents on a PEC F100 by IE-DDM

\section{CONCLUSION}

This paper presents a domain decomposition method using integral equations for the scattering of metallic targets and permitting to study the antenna sitting on large platforms. First, the formulation of the methodology is detailed for EFIE, MFIE and CFIE allowing processing both closed and opened conducting surfaces. Numerical results show that the IE-DDM method is as accurate as a mono-domain resolution and improves the convergence.

\section{REFERENCES}

[1] Z. Peng, X. C. Wang, J. F. Lee, "Integral Equation Based Domain Decomposition Method for Solving Electromagnetic Wave Scattering From Non- 


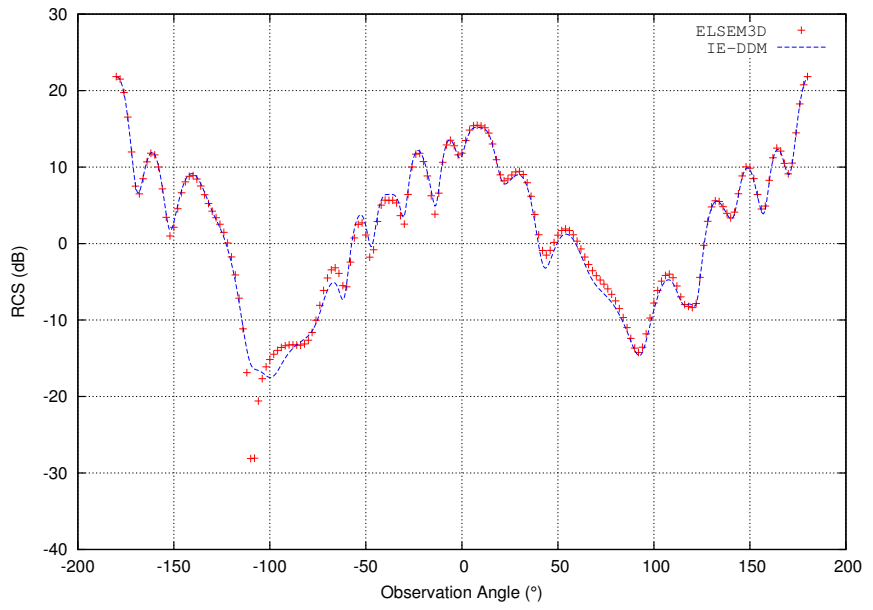

Fig. 5. Comparison of the Radar Cross Sections

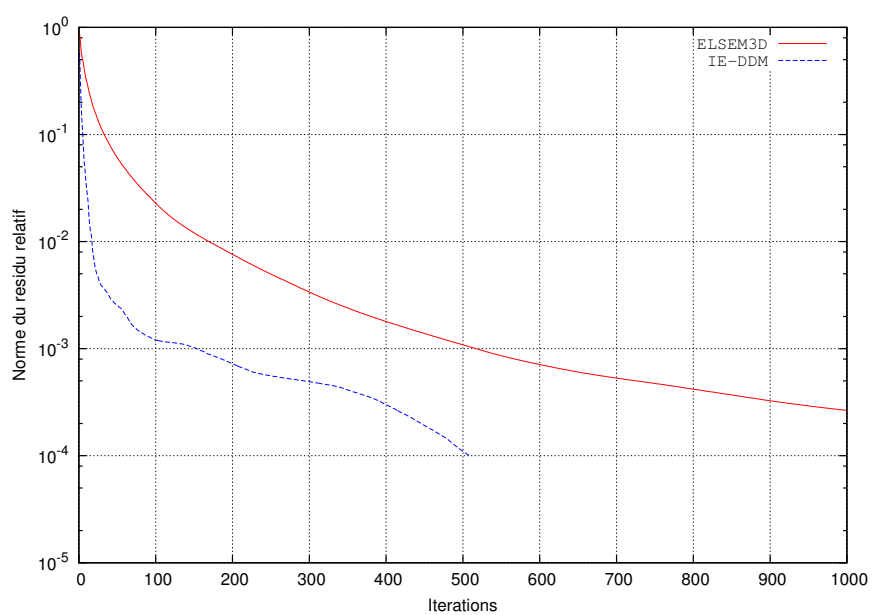

Fig. 6. Comparison of the convergences

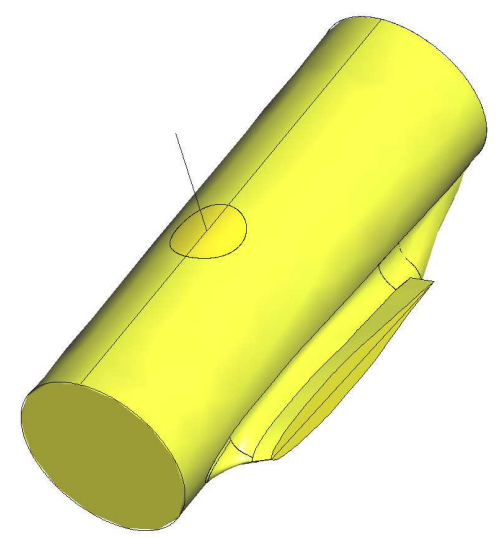

Fig. 7. Monopole antenna installed on a sub-domain

Penetrable Objects", IEEE Trans. on Antennas and Propagation, Vol.59, no.9, September 2011.

[2] J. Van Bladel, Electromagnetic Fields, 2nd ed.. The IEEE Press Series on Electromagnetic Wave Theory, 1964.

[3] S. M. Rao, D. R. Wilton, A. W. Glisson, "Electromagnetic Scattering by Surfaces of Arbitrary Shape", IEEE Trans. on Antennas and Propagation,

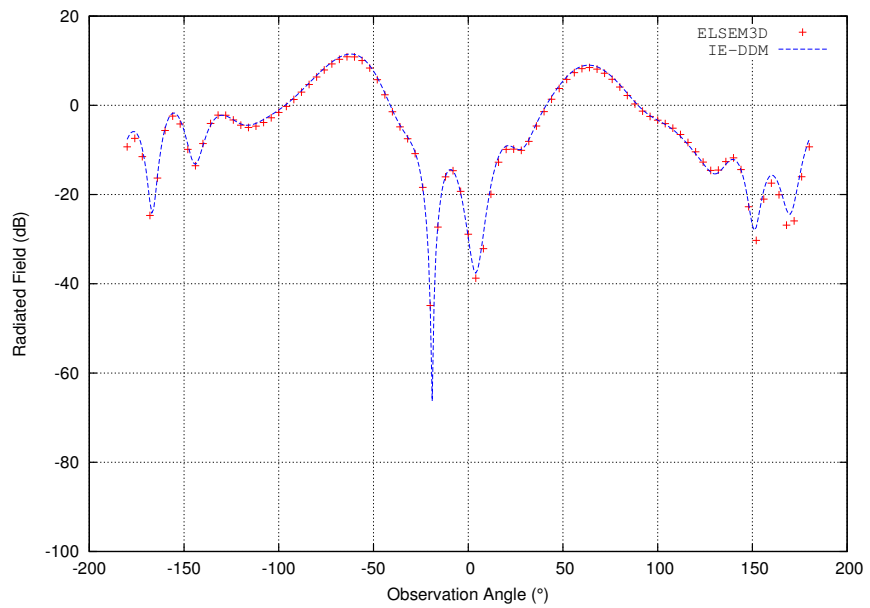

Fig. 8. Comparison of the radiation patterns

Vol. AP-30, no. 3, 1982.

[4] A. Barka, "Integration of Antennas Onboard Vehicles and Diffraction by Large and Complex Structures With Multiple-Domain-MultipleMethods Techniques", Proceedings of the IEEE, September 2012.

[5] M. Bebendorf, "Approximation of boundary element matrices", Numer Math., Vol. 80, pp. 565-589, June 2000.

[6] S. Rjasanow, O. Steinbach, The Fast Solution of Boundary Integral Equations, Springer, 2007.

[7] S. C. Eisenstat, H. C. Elman, M. H. Schultz, "Variational Iterative Methods for Nonsymmetric Systems of Linear Equations", SIAM J. Numer. Anal., Vol. 20, no.2, April 1983.

[8] J. Simon, Extension des Mthodes Multiples Rapides : Rsolution pour des seconds membres multiples et applications aux objets dilectriques, Thse de doctorat, ONERA, 2003 